\title{
CYBERBULLYING NA CONTEMPORANEIDADE: UM PANORAMA DE PESQUISAS EM EDUCAÇÃO
}

\author{
Fernanda Ribeiro de Souza, Gabriela Amorin Ferruzzi, Claudia Maria de Lima \\ Universidade Estadual Paulista - UNESP, Programa de Pós-Graduação em Educação, Presidente Prudente, SP. E-mail: \\ ferrisouza@hotmail.com
}

\begin{abstract}
RESUMO
As relações sociais presentes na sociedade contemporânea estão demarcadas pela ampliação de espaços de interação. $O$ ambiente virtual tornou-se um importante meio para comunicação e em especial, apontamos para as redes sociais na internet. No entanto, a busca por recursos digitais deu evidência a conflitos, como o cyberbullying. Deste modo, o objetivo da pesquisa foi identificar e analisar como o cyberbullying tem sido tratado em investigações científicas na contemporaneidade. Neste artigo, trazemos um panorama de pesquisas em Educação sobre o tema com base no Banco de Teses e Dissertações da CAPES. O levantamento bibliográfico do tipo estado da arte resultou em 44 trabalhos, dos quais 12 pertencem aos Programas de PósGraduação em Educação. As pesquisas identificadas estão apresentadas no texto a partir de uma breve descrição de seus objetivos. Os resultados apontam a relevância dos estudos observados e a necessidade de novas pesquisas que abordem a violência na internet.
\end{abstract}

Palavras-chave: Cyberbullying, internet, educação.

\section{CYBERBULLYING IN CONTEMPORANITY: A PANORAMA OF RESEARCH IN EDUCATION}

\begin{abstract}
The social relations present in contemporary society are marked by the expansion of spaces of interaction. The virtual environment has become an important medium for communication and in particular, we point to social networks on the internet. However, the search for digital resources has given evidence to conflicts, such as cyberbullying. Thus, the objective of the research was to identify and analyze how cyberbullying has been treated in contemporary scientific investigations. In this article, we bring a panorama of research in Education on the subject based on the Bank of Theses and Dissertations of CAPES. The state-of-the-art bibliographic survey resulted in 44 papers, of which 12 belong to the Graduate Programs in Education. The identified researches are presented in the text from a brief description of their objectives. The results point out the relevance of the studies observed and the need for new research that addresses violence on the Internet.
\end{abstract}

Keywords: cyberbullying, internet, education. 


\section{INTRODUÇÃO}

A contemporaneidade tem sido reconhecida por acomodar um uso acentuado de tecnologias digitais de informação e comunicação (TDIC). Isso permite com que muitos autores a chamem de era da informação, era do virtual, sociedade pós-industrial ou até mesmo sociedade da informação e do conhecimento (MORIGI; PAVAN, 2004).

Os indivíduos convivem nessa era com novos modos de interação e comunicação que trazem assistência para as atividades da vida cotidiana. Embora tantos benefícios possam ser identificados por meio do uso de tecnologias digitais, alguns conflitos são identificados. No mundo físico, a violência repetitiva, seja ela física ou não, contra outra pessoa é denominada de bullying (FRANCISCO; LIBÓRIO, 2011). No ambiente virtual, essa agressão recebe o nome de cyberbullying. (WENDT; LISBOA, 2014).

O cyberbullying tem uma rápida disseminação e pode aparecer em diferentes lugares e com autoria anônima. Sua divulgação abrange desde vídeos, páginas criadas com foco de agressão, mensagens constrangedoras ou outras atitudes de intimidação (ROCHA, 2010).

A violência manifesta pelo cyberbullying pode resultar em danos para a vida do indivíduo e ainda algumas dessas consequências podem permanecer arraigadas aos envolvidos durante toda a vida. Rodeghiero (2012) cita como exemplo, a insegurança e a perda da autoestima.

Todos esses fatores têm implicações para a Educação, prejuízos que podem ser visualizados na vida dos estudantes e comprometer o rendimento escolar. Neste sentido, discutir sobre cyberbullying na contemporaneidade é de fundamental relevância para uma reflexão e uma ampliação de estudos que visem uma educação de qualidade aos estudantes. Como destaca Schreiber e Antunes (2015) medidas preventivas precisam ser delineadas diante da necessidade do uso saudável da internet.

Desse modo, o objetivo da pesquisa foi identificar e analisar como o cyberbullying tem sido tratado em investigações científicas na contemporaneidade. Assim, apresentamos um panorama de pesquisas em Educação sobre cyberbullying a partir de um levantamento bibliográfico no portal do Banco de Teses e Dissertações da Coordenação de Aperfeiçoamento de Pessoal de Nível Superior (CAPES). Foram encontrados 44 trabalhos, sendo 12 pertencentes aos Programas de PósGraduação em Educação.

\section{METODOLOGIA}

Para identificar as teses e dissertações sobre cyberbullying, a pesquisa, de abordagem qualitativa, foi realizada a partir do levantamento bibliográfico do tipo estado da arte. Este tipo de estudo tem como característica particular a apresentação e discussão de produções acadêmicas de variados campos de conhecimento (FERREIRA, 2002). Para Romanowski e Ens (2006, p.39) "a realização destes balanços possibilita contribuir com a organização e análise na definição de um campo, uma área, além de indicar possíveis contribuições da pesquisa para com as rupturas sociais".

Além disso, as autoras destacam que

Estados da arte podem significar uma contribuição importante na constituição do campo teórico de uma área de conhecimento, pois procuram identificar os aportes significativos da construção da teoria e prática pedagógica, apontar as restrições sobre o campo em que se move a pesquisa, as suas lacunas de disseminação, identificar experiências inovadoras investigadas que apontem alternativas de solução para os problemas da prática e reconhecer as contribuições da pesquisa na constituição de propostas na área focalizada (ROMANOWSKI E ENS, 2006, p.39)

O acesso ao portal digital da CAPES por meio da palavra-chave "cyberbullying" resultou em 44 trabalhos, dos quais 12 estavam ligados aos Programas de Pós-Graduação em Educação. 


\section{RESULTADOS}

Os trabalhos identificados nos Programas de Pós-Graduação em Educação do banco da CAPES correspondem a nove dissertações e três teses. Todos de recente realização, variando entre 2010 e 2018.

As pesquisas serão descritas seguindo a ordem de aparição no banco da CAPES, deste modo, a primeira pesquisa: Representações Sociais do cyberbullying na mídia e na escola, trata-se de uma tese defendida no ano de 2014 por Andrea Muller Garcez. Contou com o objetivo de identificar e analisar o papel da instituição educativa diante do cyberbullying, com base na fala de diretores e coordenadores pedagógico e no discurso escolar e midiático.

Na sequência, a dissertação de Marielly Rodrigues Mandira, de 2017, traz como título: Cyberbullying entre estudantes: fatores individuais e do contexto escolar. Seu objetivo foi identificar a ocorrência de cyberbullying entre estudantes de escolas públicas e relações a fatos individuais e do contexto escolar, com base em estudo descritivo correlacional entre as variáveis de vitimização e agressão no contexto escolar e virtual.

Por detrás das telas: uma análise da postura reativa das vítimas de cyberbullying, de Thais Emilia Rodrigues Vaz, 2017, apresentou como foco a análise de material em vídeos e campanhas em combate às ações de cyberbullying, verificando deste modo, a reação dos indivíduos diante esses depoimentos.

O uso da tecnologia nos tempos atuais: análise de programas de intervenção escolar na prevenção e redução da agressão virtual, de Thais Cristina Leite Bozza, 2016, teve por finalidade, que teve como objetivo descrever e analisar programas educativos de prevenção e redução do cyberbullying e da cyber agressão, por meio de uma pesquisa bibliográfica, do tipo Estado da Arte.

A pesquisa de doutorado realizada por Ademar Alves Santos, de 2017, denominada: Cyberbullying, mídia e educação à luz do pensamento complexo, com intento de reflexão acerca das condições de produção e compreensão do fenômeno cyberbullying pela mídia, à luz do Pensamento Complexo.

A terceira tese identificada: Scraps de ódio no Orkut: cyberbullying, contextos e ressonâncias da violência virtual que atinge o professor, de Telma Brito Rocha, 2010, possuiu como intento analisar o processo de produção discursiva, bem como as ações de cyberbullying direcionadas a professores no Orkut, os modos e estratégias deste tipo de violência por meio do virtual.

A dissertação de 2015: Do bullying ao cyberbullying: história e memórias escolares (19932011), permitiu como que Silvania da Silva Santos investigasse os espaços percorridos para a construção do objeto de estudo e os diálogos de alguns autores.

A investigação de mestrado: Cyberbullying e ambiência escolar: Os adolescentes e seus professores convivendo na cultura digital, de autoria de Francieli Lorenzi Fracari Della Flora, ano 2014, forneceu subsídios para investigar como ações de cyberbullying podem repercutir no ambiente escolar, com base no pensamento e atitudes de adolescentes e seus professores.

A dissertação de Fernanda Ribeiro de Souza, denominada: Representações sociais sobre cyberbullying: a realidade de uma escola de ensino médio foi publicada em 2017 e apresentou como objetivo identificar e analisar as representações sociais de diretores, professores e estudantes acerca do cyberbullying e suas manifestações nas redes sociais.

A mais recente investigação publicada no portal, de 2018: O suporte social diante da discriminação e da vitimização na adultez emergente, de Sarah Aline Roza, teve por finalidade caracterizar relações entre dimensões que caracterizam a vitimização e variáveis sociodemográficas, assim como de experiências acadêmicas para estudantes de universidades.

A dissertação de 2016, de Angelo Caminha Munhoz: Os adolescentes e o uso do whatsapp: laços e embaraços nas suas sociabilidades, analisou como os adolescentes, de 15 a 18 anos, se 
apropriam do aplicativo de mensagens por dispositivos móveis whatsApp e quais os resultados desse meio para as suas sociabilidades.

De forma mais indireta, o último registro contido no portal da CAPES, a dissertação de 2016: Midiaeducação na prática: a experiência de uma escola jesuíta que une educação e comunicação em Curitiba, de Christiane Beller, não abordou diretamente o cyberbullying como objeto de pesquisa, sua finalidade foi analisar a repercussão das tecnologias digitais de informação e comunicação em uma escola confessional.

As pesquisas que trazem o delineamento de programas de prevenção oferecem indícios de estratégias que contribuem para reflexões relativas ao modo de estabelecimento das relações sociais na sociedade contemporânea. Na perspectiva das autoras, Tognetta e Rosário (2013) a violência tem uma ligação próxima com a falta de valores morais.

\section{DISCUSSÃO}

Os estudos identificados no Banco de Teses e Dissertações da CAPES, têm suas publicações recentes, a partir de 2010 até 2018. Dos 12 trabalhos apresentados, um se distanciou da temática, posto que, o cyberbullying não foi o foco da investigação. Apesar disso, cada pesquisa apresentou significativas análises a respeito do cyberbullying.

Alguns apresentam enfoques próximos, abordando o ambiente escolar. Há também a constatação de estudos com análise de aplicativos e redes sociais como exemplo Orkut, Facebook e Whatsapp, configurando assim, um elo com a realidade atual, visto que o cotidiano dos brasileiros é demarcado pelo uso de recursos digitais, que podem resultar de forma positiva ou negativa aos usuários (KRZYK; KUNST, 2012).

Duas pesquisas, uma de mestrado e outra de doutorado, seguem a vertente da Teoria das Representações Sociais (MOSCOVICl, 2003) e apontam para conhecimentos do senso comum sobre cyberbullying, que variaram de acordo com o grupo social.

Tendo em vista a tendência de estudo adotada por cada pesquisa, os resultados revelaram uma série de contribuições na área da educação. Na medida em que o fenômeno é compreendido, medidas preventivas podem surgir. Assim como, a possibilidade de conscientização aos leitores e incitação de projetos de diferentes âmbitos.

\section{CONCLUSÃO}

O surgimento das tecnologias digitais de informação e comunicação contribuíram para novas formas de comunicação, interação, socialização, entretenimento e também novos modos de trabalho. Fatores que facilitaram as relações estabelecidas entre as pessoas, mas que podem causar prejuízos, alguns até irreversíveis, caso não sejam utilizadas de forma consciente e reflexiva.

A educação assume um importante papel diante da violência, na medida em que pode oportunizar meios de discussão do assunto, colaborando na prevenção e redução do cyberbullying. Embora, algumas situações de violência virtual não aconteçam no interior da escola, seus efeitos alteram o cotidiano das aulas e consequentemente implicam em ações da escola.

Assim, os trabalhos decorrentes de mestrados e doutorados destacados neste levantamento oferecem passos oportunos para o delineamento de políticas públicas de caráter educacional e reflexões acerca do universo escolar que contribuem para o currículo da escola. 


\section{REFERÊNCIAS}

BOZZA, T. C. L. O uso da tecnologia nos tempos atuais: análise de programas de intervenção escolar na prevenção e redução da agressão virtual. 2016. s.n. Dissertação (Mestrado em Educação). Programa de Pós-graduação em Educação da Faculdade de Educação da Universidade Estadual de Campinas. Campinas, 2016.

FERREIRA, N. S. A. As pesquisas denominadas "estado da arte". Educação \& Sociedade, São Paulo, ano 23, n. 79, p. 257-272, ago. 2002.

FRANCISCO, M. V.; LIBÓRIO, R. M. C. Reflexões sobre a superação de concepções individualizantes sobre o bullying escolar. In: RIBEIRO, A. I. M. et al. (Orgs.), Educação contemporânea: caminhos, obstáculos e travessias. São Paulo: Cultura Acadêmica, 2011.

GARCEZ, A. M. Representações sociais do cyberbullying na mídia e na escola. 2014. 186 f. (Tese de Doutorado) Departamento de Educação. Pontifícia Universidade Católica do Rio de Janeiro. Rio de Janeiro, 2014.

KRZYK, T.; KUNST, R. Redes sociais na internet: contextualização, mercado e desenvolvimento. Cippus, v.1, n.2, p.74-97, 2012.

MORIGI, V. J.; PAVAN, C. Tecnologias de informação e comunicação: novas sociabilidades nas bibliotecas universitárias. Ciência da Informação. Brasília, v. 33, n. 1, p. 117-125, jan./abr.2004.

MOSCOVICl, S. Representações sociais: investigações em psicologia social. 5a ed. Petrópolis, RJ: Vozes, 2003.

ROCHA, T. B. Scr@ps de ódio no Orkut: cyberbullying, contextos e ressonâncias da violência virtual que atinge o professor. 2010. 211 f. Tese (Doutorado em Educação) - Universidade Federal da Bahia, Faculdade de Educação. Salvador, 2010.

RODEGHIERO, C. C. Violência na internet: um estudo do cyberbullying no Facebook. 2012. Dissertação (Mestrado em Letras) Programa de Pós-graduação em Letras. Universidade Católica de Pelotas. Pelotas, 2012.

ROMANOWSKI, J. P.; ENS, R. T. As pesquisas denominadas do tipo "Estado da Arte". Diálogos Educacionais, v. 6, n. 6, p. 37-50, 2006.

SCHREIBER, F. C. C.; ANTUNES, M.C. Cyberbullying: do virtual ao psicológico. Boletim Academia Paulista de Psicologia. São Paulo, v. 35, n. 88, p. 109-125, 2015.

SANTOS, A. A. Cyberbullying, mídia e educação à luz do pensamento complexo. 2017. 239 f. Tese (Doutorado em Educação). Universidade Nove de Julho - UNINOVE. São Paulo, 2017

SOUZA, F. R. Representações Sociais sobre cyberbullying: a realidade de uma escola de ensino médio. 2017. 197 f. Dissertação (Mestrado em Educação). Programa de Pós-graduação em Educação. Universidade Estadual Paulista. Faculdade de Ciências e Tecnologia. Presidente Prudente, 2017.

WENDT, G. W.; LISBOA, C. S. M. Compreendendo o fenômeno do cyberbullying. Temas em Psicologia. Ribeirão Preto, v. 22, n. 1, p. 39-54, abr. 2014. 